\title{
NON UNION OF FRACTURE NECK OF FEMUR FOR INTERTROCHANTERIC OSTEOTOMY
}

\author{
Singh G.P.*
}

\section{ABSTRACT}

BACKGROUND: Femoral neck fracture is a difficult problem in adult patients and without proper treatment leads to high functional impairment and frequent complications.

OBJECTIVE: The aim of this study was to see the functional outcome in patients with non union of fracture neck of femur treated with intertrochanteric osteotomy.

MATERIAL AND METHODS: All 14 cases operated and non operated (neglected) fracture neck of femur were enrolled for the study 2003 to 2009 in National Medical College, Birganj and Janaki Medical College, Janakpur. Patients were treated conservatively and in some patients failure of fixation was used to evaluate the functional outcome.

RESULTS: There were 14 patients with average age of 30 years with age range 14-50 years. 8 patients were male and six were female. 10 patients were previously treated conservatively and 4 patients presented with failure of fixation. Average duration of fracture was 7 months (2 months -3 years with pauwel angle of 32.8 .

CONCLUSION: Neglected neck of femur fracture should be treated by three cancellous screw fixation with molded and angled narrow DCP plate, valgus osteotomy and autogenous bone grafting round the fracture to achieve a high rate of patient satisfaction and satisfactory functional outcome.

KEYWORDS: Neglected fracture neck of femur, Intertrochantry osteotomy, Autogenous bone grafting.

* Professor, Department of Orthopaedics, Universal College of Medical Sciences \& Teaching Hospital, Bhairahawa, Nepal

For Correspondence

Dr. G.P. Singh, M.S., F.I.C.S. (U.S.A.),

F.A.C.S.(U.S.A.), M.I.F.S.C. (India),

Professor,

Department of Orthopaedics,

Universal College of Medical Sciences \& Teaching Hospital, Bhairahawa, Nepal

Email:gp_singh1@hotmail.com 


\section{INTRODUCTION}

Non-union fractures neck of femur in young adults is a vital problem. $^{1-4}$ from practical point of view if fracture remains untreated for 3 weeks or more it is designated as neglected fracture. As internal fixation alone has a high frequency of failure rate so in such cases to achieve union of fracture internal fixation has to be combined with some type of bone graft or osteotomy.

Due to the life style and religious requirements of the patients of western countries are treated by hip arthroplasty. The people of our country want to squat or sit in cross legged position. The movements required are not possible with total hip arthroplasty. It is therefore required by all means that the god gifted hip joint should be preserved particularly in young adults. We have used the traditional pauwel intetrochantric osteotomy, adding autogenous bone grafts taken come osteotomy site to non union site. Molded angle narrow DCP was used as requirement for non union fracture neck in adults in our study. Certain modifications have been made in this procedure.

\section{MATERIAL AND METHODS}

The study included fourteen cases of operated and non operated (neglected) cases of femoral neck fractures. In operated cases, improper fixation was the apparent cause of non-union and non operated case were treated by quack (Bone setter).

According to pauwal classification, the patients were divided in three groups. 6 under C-Arm, operations were carried out on fracture table. Directed lateral approach was used to expose the fracture site. Vastus lataralis was lifted sub periosteally after releasing it in L shape. In operated cases implant used were removed and fibrous tissue were excised at the fracture site. Narrow DCP of 06-07 holes molded round to greater trochanter and angled according to the required angle of intertrochanter osteotomy. Fracture neck reduced and fixed with initially single 6.5 concellous screws through the contoured narrow DCP. ${ }^{7}$ At osteotomy level are made proximol transeverse cut and distal angle cuts.

Pre-determined size of wedge usually of 30 degree was removed. Two more 6.5 cancellous screws through plate in the neck passed and compressed. The lower limb was abducted to align the femur to plate and reduction clamps were applied. Angled DCP was fixed to femur using $4.5 \mathrm{~mm}$ cortical screws. The removed bone was used as a graft around the fracture site anteriorly and posteriorly. ${ }^{7}$ Skin traction was applied to every case postoperatively for 04 weeks to avoid any implant failures as our patients were mostly illiterate and do not follow the instruction strictly. The patients were advised isometric leg exercise along with skin traction. After one month every patient was examined clinically, under C-Arm and radiologically to see sign of union. After two months, patients were mobilized with non weight bearing with walker. In $3^{\text {rd }}$ months if patient is pain free on passive hip movements, radiologically satisfactory position and C-Arm shows sign of union. Patient is asked to walk with partial weight bearing and once patient walked with confidence and there is no pain he/she was allowed full weight bearing. Patients were reviewed at 06 month, 09 month and 1 year after surgery individually.

\section{RESULTS}

The mean age of patients was 30 years with age range 14-50 years. Eight patients were male and six were female. Ten patients were treated by Quack, one female patient got bullet injury to neck of femur and three patients improper fixation was done at district private hospital. The duration between the injury and final surgery was 7 months on average ( 2 months to 3 years). None of the patients had any significant medical problem. Initial radiographs of the both hips were taken, to assess the degree of neck resorption, Pauwel angle and inclination of the fracture.

All the fracture showed different degree on neck resorption, but maximum resorption were found in two cases, one with bullet injury other three years old treated with DHS. No evidence of femoral head collapse was observed in any of femoral neck fracture. No special investigations were performed to detect per collapse or avascular necroses of femoral head. There were 8 Pauwel types II and 6 type III fractures in our series.

Clinically healing was presumed to be present when the patient was pain free and was bearing full weight with out any support. Radiologically healing was determined by presence of bridging trabeculae of bone across the non union site on plain radiographs and C-Arm examination. All hip movements dynamically were visualized on $\mathrm{C}$-arm examination.

One of the patient got infected after one month of surgery and girdle stone procedure was done in this case. In two cases, one with bullet injury and other 3 years old operated non union, got united after 28 weeks but follow up radiology showed subluxation of head of femur perhaps due to resorption of femoral neck. Average follow up was 18 months in 13 cases. Average time to healing of non union was 18 weeks (16-28 Weeks). One patient who got subluxation of femoral head, complained of pain at night mostly but she walked pain free with relative good range of movements. There were no thoromboembolic complications or implant failure in our series. Functional out come was based on Askin Bryan Criteria. ${ }^{8.9}$

There were 7 cases with excellent results, 5 with good and 1 case was with fair results. There was poor out come in one case that got severe hospital infection and ended up in gridlestone 
pseudarthrosis. One operated patient showed signs of non union up till 24 weeks. There patients started walking with full weight bearing with support of walker.

\section{DISCUSSION}

Femoral neck fracture in young adult is an emergency and the patient needs to be treated by early closed reduction and stable fixation. In our country late and neglected presentation of femoral neck fracture suggests that if fracture neck of femur is more than 2-3 weeks old then primary osteosynthesis with cancellous screw alone does not give good results. ${ }^{10.11}$ Meyers also classified acute fracture of neck of femur on those treated up to 30 days after injury and those beyond 30 days as delayed or non union.

The aim of treatment in young adult patients with non union or neglected maltreated fracture neck of femur without apparent avascular necrosis is salvage of God gifted femoral head.

Pauwel showed that after reduction and placing the non union of fracture neck under compression by resecting the laterally based wedge osteotomy resulted in union of fracture, ${ }^{7}$ In our procedures, we used cancellous graft taken from osteotomy site and placed around the fracture neck, and three 6.5 cancellous screws were passed through molded narrow DCP, to avoid any fracture non union of fracture neck of femur and to achieve revascularization of head of femur. Post operatively, every case was given skin traction for four week to secure fixation. Except one patient who was given skin traction for 16 weeks. We achieved union rate of $92.8 \%$. The largest series in literature is that of Marti et al. ${ }^{13}$

They presented results of valgus osteotomy in 41 patients of non union of femoral neck fractures, with union rate of $86 \%$ they experienced technical difficulties in 6 patients requiring a second surgery for re-fixation. 7 other patients required replacement due to persistent non union. Late segmental collapse or implant failure at the shaft. Angelen reported 13 patients with failed internal fixation of femoral neck fracture treated with valgus osteotomy achieving a union rate of $100 \%$.

Studies comparing blade plate with compression screw for fixation of fracture neck of femur have high rate of suboptimal position of implant, cut through and implant failure with blade plate. ${ }^{14}$ While using blade plate, hammer impact can displace the fragments. ${ }^{15}$ In dynamic hip screw, no doubt the implant is not hammered, but the chances of rotation of head of femur are there around DHS reamer as compared with application of three cancellous screw through molded narrow DCP give equal compression at fracture neck of femur, reduce the chance of cut through, and rotation of head of femur as well. The mechanism of hip joint shows that in one leg stance phase a load of at last three time body weight will transfer $21^{\circ}$ inferiolaterally. ${ }^{16}$
We as a routine planed a wedge $30^{\circ}$ as it is difficult to precisely calculate the size of wedge. This can be explained on the basis of biological as well as mechanical reasons for effectiveness of osteotomy in promoting union. ${ }^{13}$ Avascular necrosis without head collapse has not been considered to be contraindication for valgus osteotomy. Colandruccio and Anderson considered the vascular damage at the time of fracture which decides whether of not necrosis will develop. ${ }^{17}$ However Stromqust and Harriso used tetracycline and Isotope studies to show that vascular damage may be increased during the fixation of facture. ${ }^{18}$

In fresh femoral neck fractures, over correction more than 20 to 30 degree valgus of malrotation will affect the vessels and increase the chance of developing necrosis but whether this holds true in old case with resorption of neck is not clear. ${ }^{18}$ It is also well documented that revascularization of head is possible both by artery of ligamentum teres and by vessels crossing the uniting fracture. ${ }^{19}$

Pre-collapse avascular necrosis is not a contraindication for valgus osteotomy in non-united fracture neck of femur. We believe further studies by non invasive means are required to evaluate effect of osteotomy on vascularity of femoral head.

\section{CONCLUSION}

Three cancellous screw fixations with molded and angled narrow DCP plate valgus osteotomy, autogenous bone grafting round the fracture gives good outcome in neglected fracture neck of femur if done properly.

\section{REFERENCES}

1. Massic WK. fracture of the hip. J Bone and joint. 1964: 46 658-690

2. Protzman RR, Burkhalter WC. Femoral neck fractures in young adults J. Bone Joint. 1976; 758: 689-695.

3. Zolezer L, Manninger KG. Fracture of the femoral neck in adolescence injury JNagy E. 1972; 4: 41-46.

4. Kalra M, Anand M. Valgus inter trochenteric osteotomy for neglected femoral neck fracture in young adults. SICOT $2001 ; 25: 363-366$.

5. Sandhu HS, Sandhu PS, Kapoor A. Neglected fracture neck of Femur of femur apredchve classification and treatment by Osteosynthesis. Clin Orthop Relat Res 2005; 431 : 1420.

6. Pauwels F. Der Schenkelhalsbruch ein mechanics Problem: Grundlagen des Heliungsvorganges, Prognose and kausale Therapie. Stuttgart; Ferdinand Enke Varlag 1935.

7. Stewart MJ, Wells RE. JBone joint. Surg Am 1956: 33-49.

8. Sharma M, Sood LK, Kanojia RK, Alok Sud. Valgus 
Osteotomy for nonunion fracture neck femur. Ind J. Orthop 2004; $38(2): 88-91$

9. Askin SR. Bryan RS. Femoral neck fracture in young adults. Clin Orhto 1976; 114: 259-264.

10. Bout CA. Percutaneous cannulated cancellus screw fixation of femoral neck fracture. Three point Principle Injury 1997; 28: 135-139

11. Sud A. Closed Reduction and Percutaneous Cannulated Cancellus Screw Fixation of Femoral Neck Fracture. Ind J. Orthop 2000; 34: 151-152.

12. Meyers MH, Harvey JP, Moore TM. Treatment of displaced Subcapital transcervical fracture of femoral neck by muscle pedicle bone graft and internal fixation. J Bone Joint Surg Am 1933; 55:257-274

13. Marti RK, Schuller HM, Raymakers ELFB. Intertrochantric osteotomy for non-union of femoral neck. $J$ Bone Joint Surg (Br) 1989; 71: 782-787.
14. Doppelt SH, The sliding compression screw: Todays best answer for stabilization for Intertrichantrchip fracture. Ind JOrthop 2000; 34: 151-152.

15. Wu et al. Treatment of femoral neck nonunion with a sliding compression screw: comparison with and without subtrochantric valgus osteotomy. Injury. 1999; 46: 312-317.

16. Pring D. Biomechanics of hip. In: Barrett D. Essential basic science for orthopaedics Oxford: ButterwothrHeinemann. 194; 62-93.

17. Calandruccio RA, Anderson WE. Post fracture AVN of femoral head: Correlation of experience and clinical studies. Clin Orthop. 1980; 152: 49-84.

18. Stromquist B, Harrison LJ. Femoral head vitality after femoral neck fractures- Comparison between per and perioperative tetracycline labeling. Arch Orthop Trauma Surg. 1983; 101-251.

19. Catto MA. Transcervical fractures. J Bone Joint Surg (Br) 1965; 47:749-776.

\section{Universal College of Medical Sciences and Teaching Hospital is having following post graduation programmes:-}

1. General surgery

2. Paediatrics

3. Orthopaedics \& Trauma

4. Radio-diagnosis

5. Anaesthesiology

6. Psychiatry

7. Pathology

8. Clinical Anatomy
9. Internal Medicine

10. Obstetrics \& Gynaecology

11. ENT

12. Dermatology

13. Ophthalmology

14. Microbiology

15. MDGP (General Practice)

16. MD Clinical Pharmacology 